\title{
"FRCEF: The New Friction Reduced and Coupling Enhanced Finger for the Awiwi hand"
}

\author{
Werner Friedl, Maxime Chalon, Jens Reinecke and Markus Grebenstein \\ Institute of Robotics and Mechatronics, German Aerospace Center (DLR), Wessling, Germany \\ E-mails: \{Werner.Friedl, Maxime.Chalon, Reinecke.Jens, Markus.Grebenstein \}@dlr.de
}

\begin{abstract}
The combination of tendon driven robotic fingers and variable impedance actuation in the DLR hand arm system brings benefits in robustness and dynamics by enabling energy storage. Since the force measurement and motors are in the forearm the tendon path should have low friction for accurate movements and precise finger control. In this paper an enhanced generation of the Awiwi hand finger design is presented. It reduces the friction in the actuation system about 20 percent and increases the maximum fingertip force about 33 percent. A test finger was designed to evaluate different tendon couplings and to test a magnetic sensor to measure the joint position. In a next step a new finger design for DLR hand arm system has been developed. Finally, the low friction and the robustness are proven using several experiments.
\end{abstract}

\section{INTRODUCTION}

In the most recent years of humaniod robotics, research labs that are designed to suit the robot capabilities [1] are replaced by more realistic environments or even the real world [2], [3], which brings humanoid robots much closer to work with humans. This step consequently led to a paradigm change that can be seen on various robots providing enhanced robustness [4], [5], [6], [7]: Many robots nowadays are able to deal with impacts instead of trying to avoid any collision. Collisions are 'a part of the game'. The robustness of robotic hands is especially important since it is a very exposed and filigree part. A robot hand has to provide the dynamics and sensitivity to perform complex and delicate manipulation tasks while maintaining the robustness needed to interact with the real world. Thus, we developed an anthropomorphic hand for the DLR Hand Arm System [5] called the Awiwi hand [8], [9] (Fig. 1) to provide anthropomorphic:

- Robustness

- Performance

- Small size

In the recent years some remarkable newer developments have been presented that share the goals of the Awiwi hand and its redesign. Synergies can be used to achieve a tradeoff between the number of drives and the grasping performance [6].This hand is equipped with dislocatable joints like the Awiwi hand, to provide robustness against impacts/ collisions (cf. Fig. 2). The Sandia hand uses a modular concept with completely dislocatable modular fingers to provide robustness but still dexterous grasping abilities [10], [11].

The Awiwi hand is able to approach all hand postures

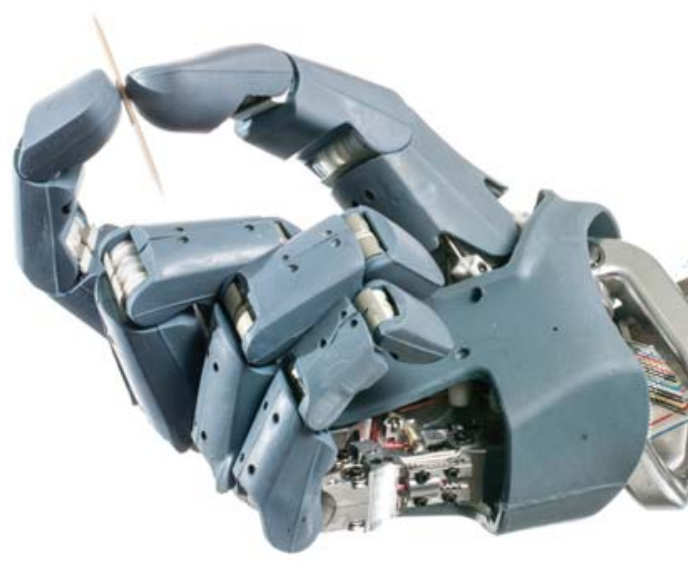

Fig. 1: The Awiwi hand

of the taxonomies presented in [12] and [13], as well as to keep an object firmly grasped even during an impact [14]. ${ }^{1}$ Furthermore, it is able to withstand the impact of a $500 \mathrm{~g}$ hammer, while in full operation, without any damage [14]. A complex system, such as the Awiwi hand, is continuously improved in order to reach always more ambitious goals. This paper presents the latest achievements in the redesign process that is carried out to enhance the following aspects:

- Improve grasping and manipulation performance

- Reduce the complexity of the system

- Reduce maintenance time and effort

The precise control of the fingers is paramount into achieving the best grasping performance. Thus, the redesign efforts are aiming at simplifying and improving the positioning accuracy and the torque sensing capabilities. Two major aspects of the hand are identified as being limitations regarding the performance of the hand controller: The friction in the guiding and the creeping of the tendons. First, the tendon forces acting on the joints must be estimated since friction of the tendon path is not directly observed and the forces are measured via the deflection of the elastic elements directly next to the actuator as discussed in [15]. Moreover, the joint friction itself is not observed, and depends on many parameters such as the temperature, the surface quality

\footnotetext{
${ }^{1}$ The impact experiment consists in hammering the grasped object with a mass of $750 \mathrm{~g}$ at a speed of $4 \mathrm{~m} / \mathrm{s}$
} 


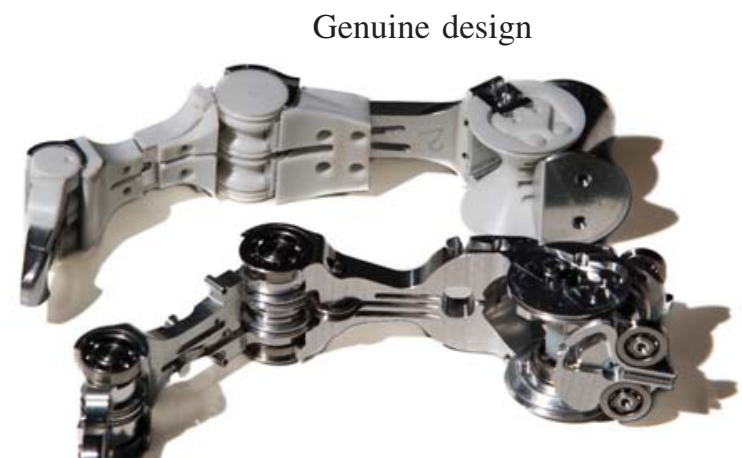

New design

Fig. 2: Initial (top) and new (bottom) finger design. Both fingers have dislocatable joints to enhance the robustness against lateral collisions. In the new design, the sliding surfaces of the initial design are replaced by ball bearings to reduce joint friction.

and the tendon pretension. Those two friction sources are limiting the torque sensitivity in the fingers, thus impairing the performance of the compliance controller. The second problem is the creeping of the Dyneema ${ }^{\circledR}$ tendons, that is, their plastic deformation over time. The joint positions are estimated based on the assumption of a rigid kinematic coupling. Therefore, as a result of the creep, the estimation of the joint position is degrading over time. The effect is most visible when the tendons are loaded asymmetrically, either due to the tendon pre-loading or the forces exerted on a grasped object.

The initial choice of the Dyneema ${ }^{\circledR}$ was motivated by the work of Friedl [15] that showed that for the small pulley diameters used in the Awiwi hand, the lifetime (in load cycles) of a Dyneema ${ }^{\circledR}$ tendon is 100 times higher than a steel tendon with an equivalent diameter. The material being non conductive is also a significant advantage over metallic cables. Unlike closed loop mechanism, antagonistic designs (underactuated or not) can handle the creep by controlling the tendon force or by introducing springs as extensors, e.g. [16]. ${ }^{2}$ The Awiwi hand uses an antagonistic $2 \mathbf{N}$ design and, therefore, is able to compensate the creeping of the Dyneema ${ }^{\circledR}$ tendons. Nevertheless, if no link side sensor is used, the estimation of the link side position must rely on a precise model of the tendon creep, which introduces a layer of complexity in the control system. Moreover, establishing a reliable model is very complicated and costly since the creep of the tendon is influenced by many factors e.g.: the load, the life time and temperature. A link side sensor allows to circumvent that issue but introduces a new set of cables and also reduces the mechanical robustness of the fingers.

The first section of the paper is analyzing the limitations of the current finger design, which was presented in detail in [9], [8], [14]. The second section highlights the design changes made to achieve a better grasping performance,

\footnotetext{
${ }^{2}$ Compensating the creep does release the spring and is therefore limited by the maximum spring travel
}

mostly achieved by significantly reducing the friction, in particular in the joints. It describes the introduction of link side sensors used to directly obtain precise angle measurements that are used as ground truth reference for the experiments. Furthermore, it describes several mechanical changes that reduce the complexity of the system and at the same time improve the performance of the Awiwi hand. Finally, an outlook to the changes planned for the complete hand is given. The third section reports the results of several experiments that confirm the effectiveness of the new design. It is followed by a conclusion and the outlook.

\section{ANALYSIS OF THE GENUINE DESIGN}

Implementing a compliant controller on robotic hands is beneficial, since it compensates uncertainties, when interacting with objects. Several approaches are available but they all require joint torque control, either directly as in the case of impedance control or indirectly in the case of admittance control. Although many methods exist that can deal with frictional effect, a very low joint and tendon friction allow to identify possible implementation errors, analyze the controller performance and gain confidence in the control architecture. It motivated the use of steel cables and ball bearings. The resulting reduction of the robustness, the reduced life time and the less available maximum forces are not a concern in that phase. Once, the controller concepts are proven, the robustness of the original design, that is bound to measurement inaccuracies, can be reclaimed with the help of skin type sensors.

The analysis is decomposed in three sections. First, the friction induced by tendons and their pulley guiding is investigated. Next, the joint friction, that is the friction due to sliding of the bone heads, is analyzed. The last part concentrates on the sliding friction between the tendons and the finger structure.

\section{A. Tendon Friction within the Forearm}

The Awiwi hand has been designed assuming that the friction within the forearm would be negligible since all guidings and pulleys used ball bearings. However, during the implementation of the controllers, it appeared that the friction was larger than expected. The total friction along the tendon path in the forearm results from the guidings in the flexible adjustable spring mechanism located in the forearm [15], the wrist pulleys ${ }^{3}$, the palm guidings with pulleys or sliding surfaces and the finger sliding surfaces. In order to investigate futher the source of the discrepancies, a calibrated weight has been lifted in a triangular motion to measure the overall friction along the complete tendon path from the winder to the palm (Fig. 3). The experiments are performed on a dedicated tensile testing machine as in [17]. The results for different materials are reported in Fig. 4. According to a standard ball bearing catalog, the expected friction of a pulley is in the order of $1 \%$. As it can be seen in Fig. 4, the internal litz wire friction of the tendon itself

\footnotetext{
${ }^{3}$ pulley is always referring to a pulley mounted on a ball bearing
} 


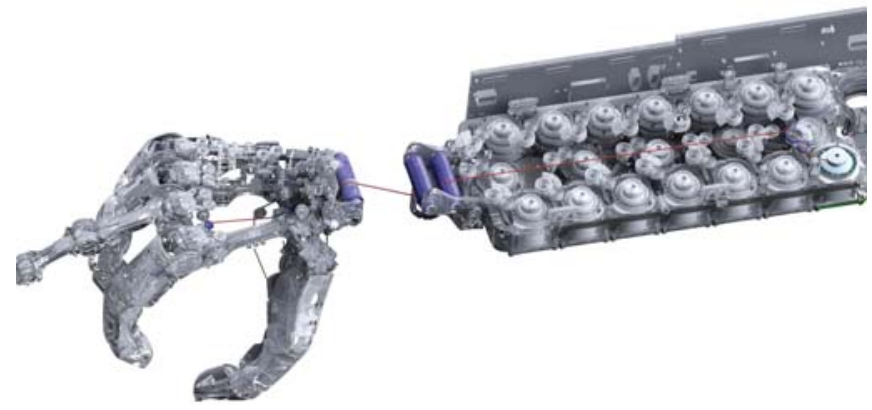

Fig. 3: Tendon routing from the winder to the base joint of the middle finger. The seven pulleys involved are displayed in purple. The friction losses can reach 30 percent if Dyneema ${ }^{\circledR}$ tendons are used.

adds a significant amount of friction. Steel cables do not introduce such a large additional friction and thus reduce the overall friction significantly. It results in a better control performance and requires less control effort for a equivalent finger behavior.

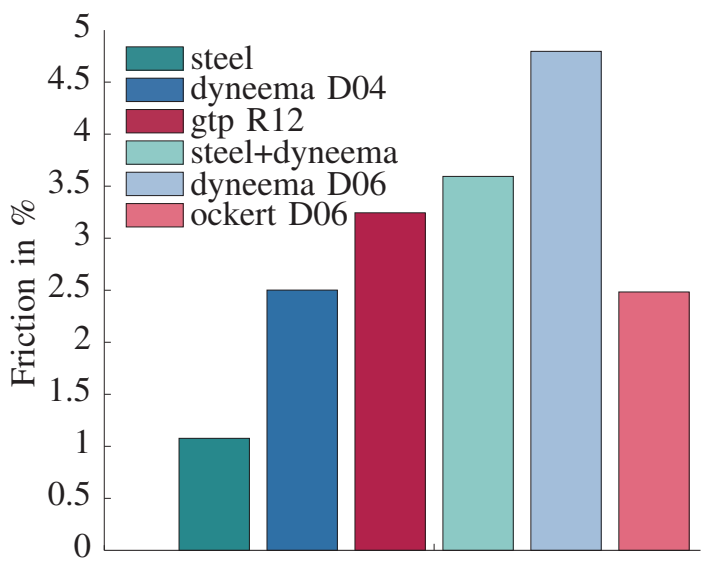

Fig. 4: Friction introduced by the pulleys for different tendon materials. A weight of $1.89 \mathrm{~kg}$ is lifted in a triangular motion on a tensile testing machine. The weight is connected with a tendon to a force sensor. Noteworthy is that steel has the lowest friction.

\section{B. Joint friction}

Particularly in the most proximal joints, the friction is significant at high tendon loads as described in [14]. This friction can harm the control performance if it is higher than estimated. Due to several factors described in the following this is partially true for the Awiwi hand. On one hand, any additional friction along the tendon path is more critical than initially expected due to the much higher friction at the pulleys. On the other hand, in particular the hyperboloids of the base joint turned out to have a significantly higher friction coefficient than expected from the first measurements and data sheets. At higher tendon loads the deformation of the hyperboloids leads to increased friction diameters ${ }^{4}$ and

\footnotetext{
${ }^{4}$ the average diameter of the sliding surface
}

the friction coefficient of the used bearing plastics increase at high contact pressures. The base joint friction can be estimated conservatively by assuming all tendon forces act in the same direction. ${ }^{5}$ The active force $\mathrm{f}_{\mathrm{t}, \text { act,joint }}$ is the tendon force needed in joint $i$ to exert an external force. It acts at the joint pulley of joint $i$ and has to overcome the friction in the joint surface caused by the normal force $\mathrm{f}_{\mathrm{n}_{i}}$. Neglecting capstan friction the normal force at joint $i$

$$
\mathrm{f}_{\mathrm{n}_{i}}=2 \mathrm{f}_{\mathrm{t}, \text { pre }}+\mathrm{f}_{\mathrm{t}, \text { act,joint }}{ }_{i}
$$

where $f_{\mathrm{t}, \text { pre }}$ is the tendon pretension force of joint $i$. The tendon loads of all more distal joints introduce an additional normal force in the respective joint. Hence, the summed up normal forces $\mathbf{f}_{\mathrm{N}_{i}}$ at joint $i$ becomes

$$
\mathbf{f}_{\mathrm{N}_{i}}=\sum_{j=i}^{n} \mathbf{f}_{\mathrm{n}_{j}}
$$

The vector $\mathrm{f}_{\mathrm{n}}$ of all joint normal forces is multiplied with a lower triangular matrix of ones $\in \mathbb{R}^{n \times n}$ in order to yield the vector of all normal forces

$$
\mathbf{f}_{\mathrm{N}_{i}}=\left(\begin{array}{ccc}
1 & \ldots & 0 \\
\vdots & \ddots & \vdots \\
1 & \ldots & 1
\end{array}\right) \mathbf{f}_{\mathrm{n}}
$$

Using (1) and the friction torque of each joint, that is $\tau_{\mathrm{fr}_{i}}=$ $\mathbf{f}_{\mathrm{N}_{i}} \mu r_{i}$, the resulting friction torque vector is

$$
\tau_{f r_{i}}=\mathbf{f}_{\mathrm{N}_{i}} \mu \mathbf{r}_{i}=\left(\begin{array}{ccc}
1 & \ldots & 0 \\
\vdots & \ddots & \vdots \\
1 & \ldots & 1
\end{array}\right)\left(2 \mathbf{f}_{\mathrm{t}, \text { pre }}+\mathbf{f}_{\mathrm{t}, \text { act,joint }}\right) .
$$

A joint friction torque $\tau_{f r b a s e}=24 \mathrm{Nmm}$ is measured for $20 \mathrm{~N}$ pretension of every tendon, a joint friction coefficient $\mu_{\mathrm{c}}=0.1$, a friction radius $r_{\mathrm{c}}$ of $3 \mathrm{~mm},{ }^{6}$ and without any external load. It represents less than $1 \%$ of the maximum joint torque of $3444 \mathrm{Nmm}$ of the index and middle finger. In the worst case scenario, the friction radius increases to the maximum hyperboloid radius of $8.2 \mathrm{~mm}$ due to bending of the hyperboloid and the per tendon pretension force is $100 \mathrm{~N}$. For this worst case scenario, the friction gets up to $656 \mathrm{Nmm}$ which is $\approx 20 \%$ of the maximum joint torque. A more detailed estimation of the friction is provided in [14]. To measure the joint friction the base joint has been mounted on a testbed. One hyperboloid was turned by a direct drive motor, while the second one has been loaded in a well defined way by a spring with a defined pressure. The friction torque $\tau_{\text {fr,base }}=170 \mathrm{Nmm}$ with a pretension of $8 \mathrm{~N}$ on every tendon. The high friction can be explained by manufacturing tolerances. Using the same method, a less significant increase in the joint friction is found for the more distal joints, most likely due to machining inaccuracies. Consequently, the reduction of the base joint friction is a design goal for the new finger design.

\footnotetext{
5 angular attachment of the tendons just adds structural/internal forces

${ }^{6}$ which is equal to the radius of the genuine design
} 


\section{Capstan friction}

The DIP tendons have the highest enlacement angle of all tendons since they have to cross the three joints of the finger [14]. Consequently, the tendon friction is maximal for the DIP tendon (Fig. 5 and (Fig. 6)). Additional friction is introduced by the two guiding surfaces required to keep the tendons in place. The sliding surfaces are manufactured with a dedicated bearing plastic in order to reduce the capstan friction and the surface wear. The efficiency of the tendon path $\mu_{\text {dip }}$ is

$$
\begin{gathered}
f_{\text {output }}=\mu_{\text {dip }} * f_{\text {Input }} \\
\mu_{\text {dip }}=\left(2-e^{\left(\left|\delta_{1}-\phi_{1}\right|\right) \mu}\right)\left(2-e^{\left(\left|\delta_{2}-\phi_{2}\right| \mu+2 \mu_{\text {static }}\right)}\right)
\end{gathered}
$$

The equation is composed of three parts, corresponding to

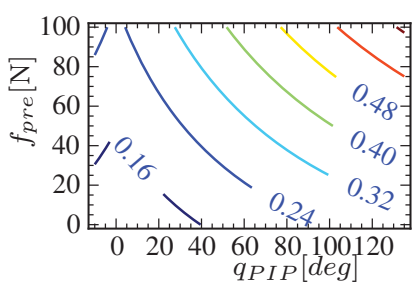

(a)

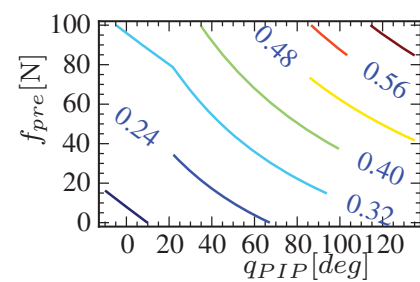

(b)
Fig. 5: Comparison of overall DIP friction with respect to PIP joint angle $q_{P I P}$ and tendon preload $f_{\text {pre }}$ using different tendon routing concepts.(a) Parallel tendons. A flexion wraps all extensor tendons. (b) Crossed tendon routing. The tendons alternatively are wrapped or unwrapped by flexion of all joints. The overall friction is lower for the parallel routing since the tendons do not have 'offset angles'. The friction of the crossed routing is dependent on the pretension for low joint angle since, in such a case, the flexor tendon is unwrapped.

the Euler tendon friction on the three joints. The adduction angle of the metacarpal joint is denoted $\phi_{1}$. The base flexion angle and the PIP flexion angle are denoted $\phi_{2}$ and $\phi_{3}$. The offsets, $\delta_{1}, \delta_{2}$ and $\delta_{3}$, describe the contact angle on the sliding surface at angle zero.

\section{Guidelines for a new design}

The following guidelines proposed for the new finger design to improve the performance of the Awiwi hand:

- The joints of the fingers should be dislocatable to protect them from overloaded

- The finger must work with steel or plastic fiber tendons for a wide operation spectrum

- All joints should have ball bearings to reduce joint friction

- The tendon routing should be optimized to reduce the maximum tendon force (steel tendon)

- A link side sensor should be integrable for future use

- Replacing a tendon should not require dismounting other parts

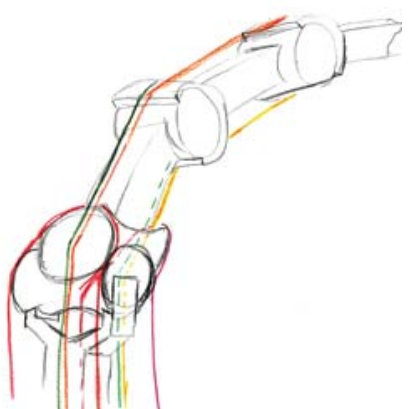

(a)

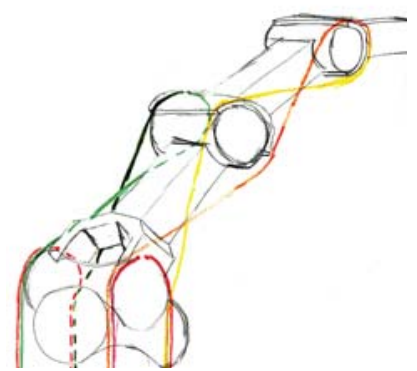

(b)
Fig. 6: Two tendon routing concept sketches: (a) Parallel tendon routing. The change in tendon length accumulates during flexion, thus requiring more control action. (b) Crossed tendon routing. The change of length of the finger end joint (DIP) tendons is canceled by the flexion of the finger middle joint (PIP). There is no relative motion between the guiding in the PIP and DIP tendons if both joint are flexed simultaneously.

- The design should integrate the inclination and twist of the genuine design

- A $N+2$ design should be used for the little and the ring finger ${ }^{7}$

\section{FRICTION REDUCED FINGER DESIGN}

A test finger has been designed to satisfy, as closely as possible, the guidelines. One half of a forearm is used, together with a special adapter, to hold and actuate the new finger prototype (cf. Fig. 7). Using the same testbed(forearm) for the single finger and the full hand is lowering the risk of an imprecise friction approximation. The finger joints

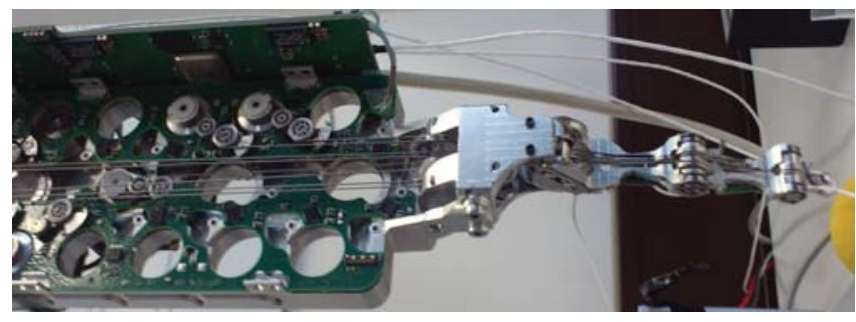

Fig. 7: One finger testbed. An adapter is used to directly mount the finger to the forearm. The tendons and the spring mechanism are clearly visible in the forearm. Unused motors are not mounted.

are equipped with ball bearings to reduce joint friction. The joints can dislocate if they overloaded, similar to the genuine design. However, unlike the genuine design, the bearings are hold by elastic clips. It maintains the bearings into the joint sockets which greatly simplifies the assembly and repair process. The clip mechanism provides a lateral

\footnotetext{
${ }^{7} N+2$ design means, if you have 4 dofs than you need 6 tendons. And you get 2 dofs of stiffness variantion
} 
stability that is not anymore provided by the tendons since the tendons are routed next to the median plane of the finger. The torque required to dislocate the joint is $54 \mathrm{Nmm}$ for the distal joint without tendon pretension. The value is a good compromise between stability during light grasps and robustness under impact. The difference in the efficiency for the distal joint between the old and the testbed design is shown in Fig. 8. The main improvement in terms of friction is due to joint between the proximal and distal phalanxes. The two distal tendons are routed over a ball bearing pulley in the proximal joint. Additionally, all sliding surfaces in the distal and proximal joints are removed. The efficiency of the distal joint is increased by $23 \%$ in a stretched out configuration. In a flexed configuration with a base flexion of $90^{\circ}$, the improvement reaches $53 \%$. A comparison of the efficiency of the genuine and new design depending on the deflexion angle is reported in Fig. 8. A pulley guiding for the PIP and DIP tendons in the base joint is too large and complex to fit. Therefore, it is not possible to further reduce the configuration dependent friction in the metacarpal joint guiding.

For the metacarpal joint the sliding surface were redesigned by pulleys. Beginning at $20^{\circ}$ in abduction a sliding surface prevent a jump out. To be able to work with steel and plastic fiber tendons the tendon fixation is formed like an undercut expanding anchor. Under pretension the tendon is automatically hold down. Additionally, a terminal screw can fix the steel tendon. For the metacarpal joint a steel clamp can be mounted to prevent the jump out of the steel tendon. Steel tendons have a behavior like a spring and therefore they want to go back without pretension to a straight line. If steel tendons are used, the maximum force should not exceed $120 \mathrm{~N}$, this is the elastic limit for bending. The maximum tensile force is double so high. If we considered the initial coupling matrix ${ }^{8}$.

$$
\mathbf{P}_{\text {index,old }}=\left(\begin{array}{cccc}
R_{1} & R_{1} & 0 & 0 \\
-R_{1} & R_{1} & 0 & 0 \\
R_{1} & -R_{1} & 0 & 0 \\
R_{1} & -R_{1} & 0 & 0 \\
0 & 0 & R_{2} & 0 \\
0 & 0 & -R_{2} & 0 \\
0 & 0 & -R_{3} & R_{3} \\
0 & 0 & R_{3} & -R_{3}
\end{array}\right)
$$

And calculated the fingertip force for $120 \mathrm{~N}$ on all flexor tendons we get.

$$
f_{\text {fingertip }}=\frac{\tau}{r_{\text {momentarm }}}
$$

The joint torque can be calculated by:

$$
\tau=\mathbf{P}_{\text {index }} \mathbf{f}_{\text {tendon }}^{T}
$$

Due to the cross coupling between proximal and distal joint the fingertip force is only $3.8 \mathrm{~N}$ for the proximal joint. If the forces are balanced for the two joints to get maximum

\footnotetext{
${ }^{8}$ The coupling between metacarpal joint and proximal and distal joints is neglected
}

fingertip force, it can reach $9 \mathrm{~N}$. If the cross coupling is replaced with a direct coupling, the maximum fingertip force rises to $21 \mathrm{~N}$. A fingertip force of $40 \mathrm{~N}$ can be reached with Dyneema tendons as flexors and a $250 \mathrm{~N}$ maximal tension.

$$
\mathbf{P}_{\text {index,new }}=\left(\begin{array}{cccc}
R_{1} & R_{1} & 0 & 0 \\
-R_{1} & R_{1} & 0 & 0 \\
R_{1} & -R_{1} & 0 & 0 \\
R_{1} & -R_{1} & 0 & 0 \\
0 & 0 & R_{2} & 0 \\
0 & 0 & -R_{2} & 0 \\
0 & 0 & R_{3} & R_{3} \\
0 & 0 & -R_{3} & -R_{3}
\end{array}\right)
$$

In figure 9, the three different tested coupling are shown. Another important point was the $\mathrm{N}+2$ design for the ring and little finger.

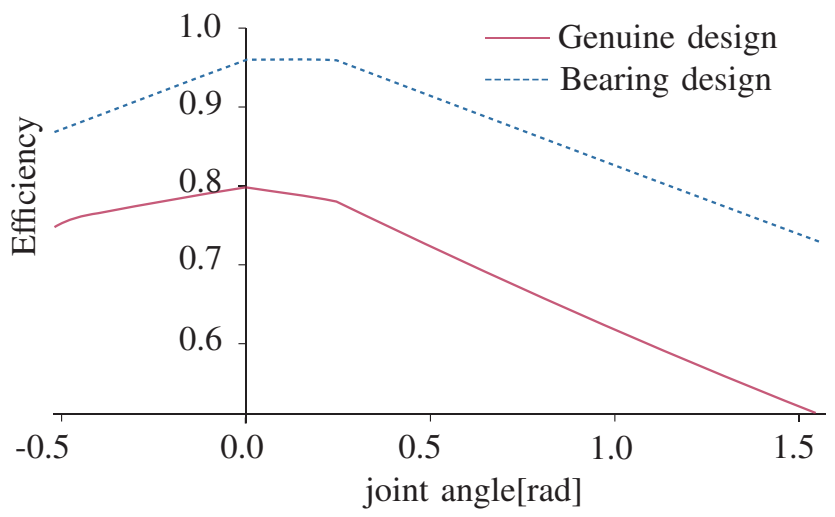

Fig. 8: Efficiency comparison between the tendon path of the genuine design and new design.

\section{A. Under Actuated Design}

In the initial design, a fixed cross coupling was used for the ring and fifth fingers. The disadvantages are: lower robustness in the distal joint, the need for a pretension mechanism for the coupling between PIP and DIP and the loss of a degree of freedom, which reduce the adaptability to grasped objects. The two DOFs of the base joint can be actuated

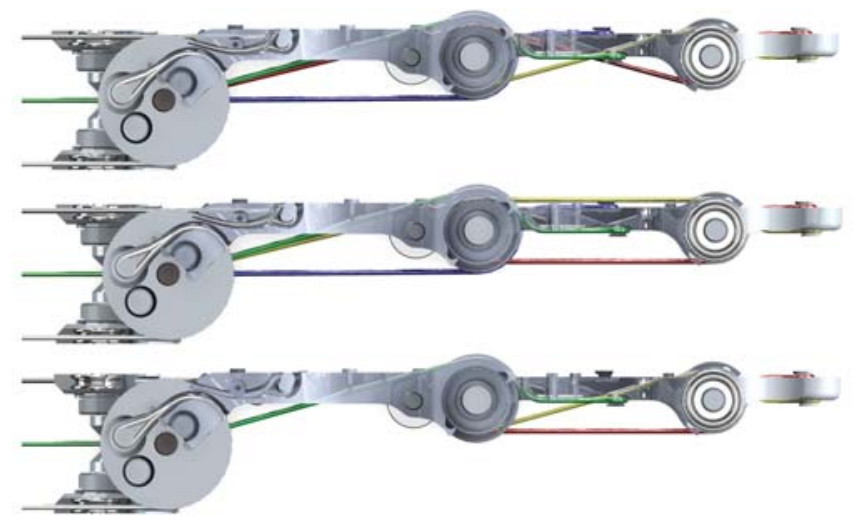

Fig. 9: Top down: cross coupling, direct coupling and $\mathrm{N}+2$. Color code: Grey metacarpal, blue proximal extensor, green proximal flexor, red distal flexor, yellow distal extensor 
with a minimum of three tendons. The single extensor runs in the middle over a sliding surface to the proximal bone. Hence, the stiffness behavior in metacarpal flexion direction is asymmetrical, because we have two flexors and only one extensor with the same maximum tendon force. The spared tendon can be used for the distal joint. According to [18] a tendon driven mechanism with active tendons is controllable only, if there exits a $\xi$, that fulfills

$$
\mathbf{f}_{\mathrm{b}}=\mathbf{A}_{2} \xi>0, \text { with } \mathbf{A}_{2}=-\left(\mathbf{J}_{j}^{T}\right)^{+} \mathbf{J}_{j}^{T}
$$

In which $\mathbf{f}_{b}$ is the bias force vector and $\left.\left(\mathbf{J}_{j}^{T}\right)^{+}\right)$is the MoorePenrose pseudo-inverse of $\mathbf{J}_{j}$. If we take the direct coupling from the index finger Fig. 10 a) and consider only the DIP and PIP joint and save the PIP extensor tendon, we get this coupling matrix for $R_{2}$ and $R_{3}$ equal one.

$$
\mathbf{J}_{\mathrm{j}}=\left(\begin{array}{cc}
R_{2} & 0 \\
R_{2} & R_{3} \\
-R_{2} & -R_{3}
\end{array}\right) \quad \text { and } \quad \mathbf{f}_{\mathrm{b}}=\left(\begin{array}{c}
0 \\
0.7 \\
0.7
\end{array}\right)>0
$$

The calculation shows that the intuition can be misleading. Indeed, it is impossible to control all joints although the mechanism seems functional. A simple test confirmed the calculations. The position of distal and proximal joints cannot be controlled. A systematic approach allows to formulate the next possible coupling (cf. Fig. 10 b)).

$$
\mathbf{J}_{\mathrm{j}}=\left(\begin{array}{cc}
R_{2} & 0 \\
-R_{2} & R_{3} \\
-R_{2} & -R_{3}
\end{array}\right) \quad \text { and } \quad \mathbf{f}_{\mathrm{b}}=\left(\begin{array}{c}
0.8 \\
0.4 \\
0.4
\end{array}\right)>0
$$

This second coupling is functional, as expected from the matrix rank. However, the coupling is not ideal w.r.t. the maximal fingertip force since the two distal tendons are pulling against the proximal tendon. This disadvantage can be corrected by using the mirrored coupling given by:

$$
\mathbf{J}_{\mathrm{j}}=\left(\begin{array}{cc}
-R_{2} & 0 \\
R_{2} & R_{3} \\
R_{2} & -R_{3}
\end{array}\right) \quad \text { and } \quad \mathbf{f}_{\mathrm{b}}=\left(\begin{array}{c}
0.8 \\
0.4 \\
0.4
\end{array}\right)>0
$$

This coupling, depicted in Fig. $10 \mathrm{c}$ ), allows the ring finger to reach a fingertip force of around $16.5 \mathrm{~N}$ with steel tendons. All other possible couplings result in a weaker finger or in an impossible mechanical design.

\section{B. Sensor}

An axial sensor [19] or a special sensor [20] can be used to get the link side position of the fingers. Both alternatives require a large space and result in a bulky design. The use of a compact analog sensor implies that extra care must be taken to implement the data acquisition electronics. An offaxis sensor on the top of the finger offers free space on the grip side and is well protected by the bones. The AS5510 from austriamicrosystem provides a small 10 bits sensor with integrated digital $\mathrm{I}^{2} \mathrm{C}$ communication. The concept requires to design a specific magnet for each joint. The test finger uses several independent electronic boards which, in a complete design, could be chained to spare wires. The position of the electronic boards is depicted in Fig. 11. Some preliminary
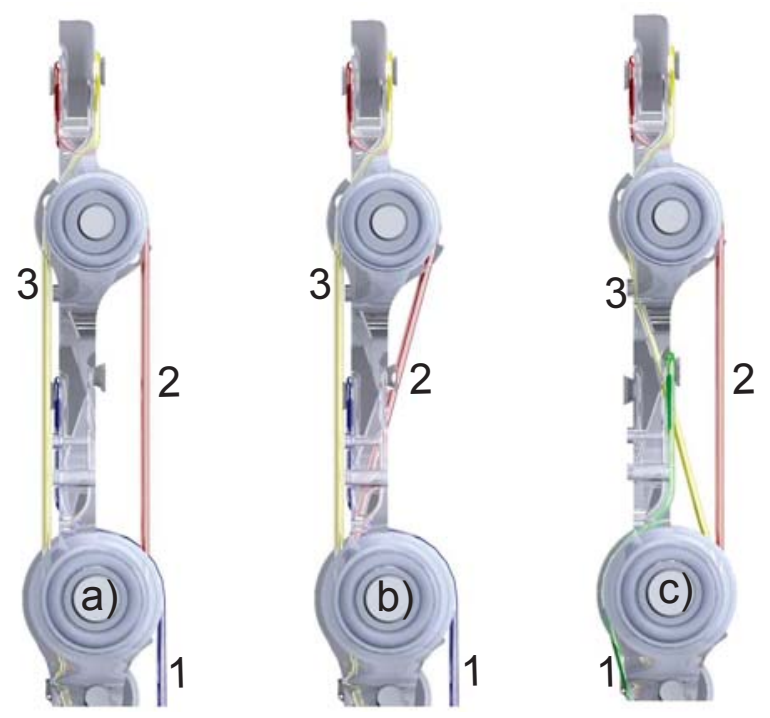

Fig. 10: Three different tendon routings for the ring finger

measurements confirmed the validity of the magnetic FEM (Finite Element Method) analysis. However, the position measurement is noisy due to the weak field in plastic bond Neodym ${ }^{9}$. A resolution of $0.3^{\circ}$ is obtained if the magnet creates the simulated field of $50 \mu \mathrm{T}$.

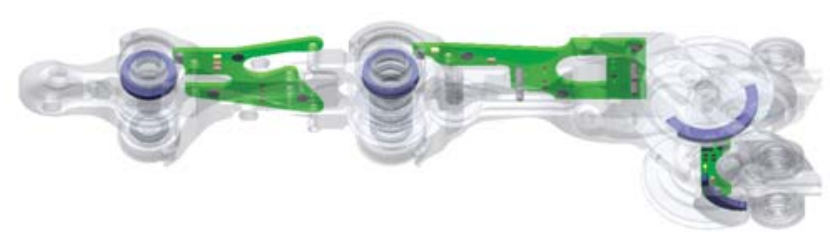

Fig. 11: Green: electronic board. Purple: magnets

\section{Fullhand}

Hence, the hand can be redesigned upon the previous analysis. The inclination and the twist of the genuine design[21] are reused in the new design Fig. 12. The fingers are designed using a parametrized model which allows to generalize the CAM (Computer Aided Manufacturing) programs. The finger parts are easily generated from parameter tables. Unlike the test finger, the end stops for the base joint are integrated to ease the zeroing procedure. The extensors of the thumb base are rolling on the base pulley. This linear coupling greatly simplifies the algorithm for the estimation of the link side position. Moreover, it enables a more accurate zeroing of the thumb base position. The new hand is designed with a place holder for the optional link side sensors. Indeed, the low friction obtained with the steel tendons should provide a sufficient accuracy. However, the sensors might be temporarily used during the control evaluation phase.

\footnotetext{
${ }^{9}$ Plastic bond magnets are cheap and easy to manufacture, however, their field is weaker than conventional magnets
} 


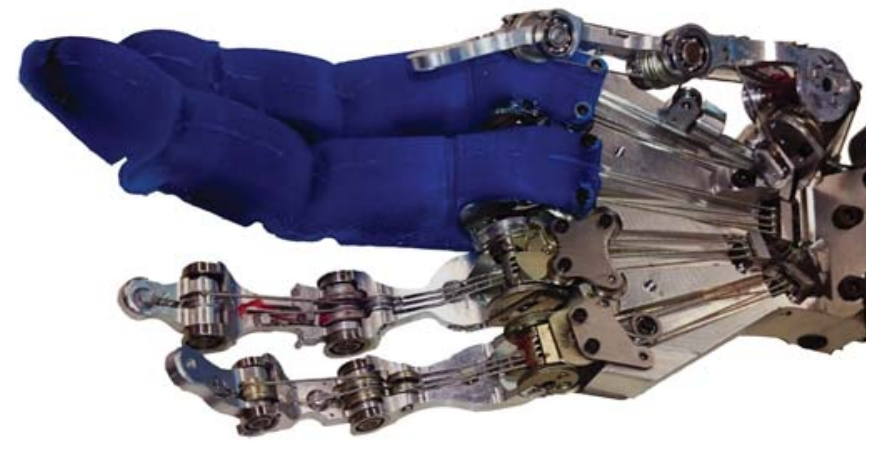

Fig. 12: View of all fingers of the Awiwi hand V2. Index finger and middle finger with mounted silicon housings.

\section{EXPERIMENTS}

\section{A. Stiffness depending accuracy}

${ }^{10}$ To give the hand user a feeling for the task depending stiffness, one important point is the repeating accuracy of the finger. Due to the progressive spring behavior we can expect a rising accuracy with higher joint stiffness. The load depending bearing friction is linear to the load and the sliding surface shows a decreasing friction with rising load. The stiffness versus torque curve has a higher order and should reduce the friction depending spring deflection.

$$
\phi_{\text {error }}=\frac{\tau_{\text {friction }}\left(f_{\text {bias }}\right)}{k\left(f_{\text {bias }}\right)}
$$

where $f_{\text {bias }}$ is the pretension of the tendon. The analysis is performed on the proximal joint. The stiffness of the distal joint is hold. The link side sensor is used as a reference. Beginning with $5 \mathrm{~N}$ pretension the bias force is rose to 65 in $15 \mathrm{~N}$ steps. The link drives a trapeze profile with a amplitude of $50^{\circ}$ (Fig.:13).

The measurements fit well between link side sensor and the result of FAS ${ }^{11}$ and motor. Only at five Newtons pretension the difference is large than 0.5 degrees. A polynom of order seven is used for the calibration of the FAS sensor which is badly conditioned at low forces. The better accuracy at low pretension can be explained by the FAS friction which is at $5 \mathrm{~N}$ higher than the joint and tendon friction. The same effect happened for $50 \mathrm{~N}$ pretension, at which only a part of the friction is compensated by the FAS. The results at the testbed are not totally transferable to the hand because in the tendon path of the full hand are more pulleys due to the palm guiding. On the other side the testbed uses not the right FAS for distal and proximal joint instead it uses the metacarpal FAS. These are softer because the spring deflection is larger for the bigger torque lever arm.

\section{B. Accuracy depending from on the tendon elongation}

Due to the creeping of Dyneema over a long load time, the link position gets lost. At the beginning of the paper three

\footnotetext{
${ }^{10}$ The accuracy behavior for different tendon materials was not tested on the finger, because the friction tests were done on a tensile machine [17].

${ }^{11}$ Flexible antagonstic spring. ??
}
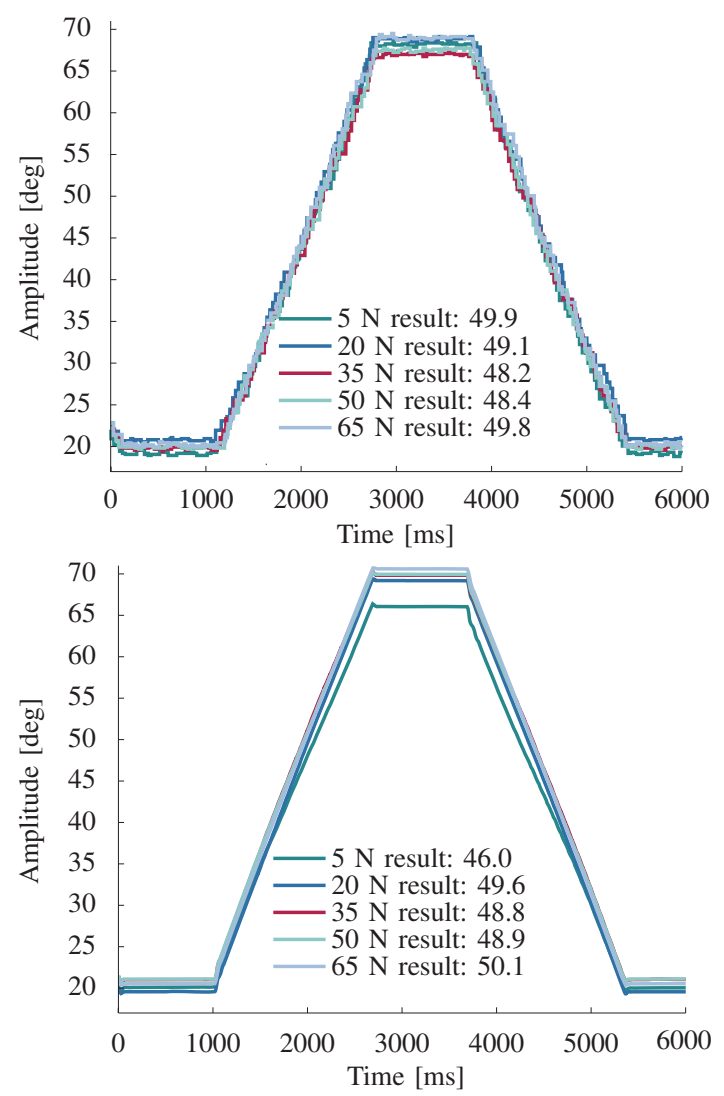

Fig. 13: Comparison of moving 50 degree desired; top: reached precision measured with link side sensor, down: reached precision measured by motor and FAS sensor

solution are shown. To compare this three the finger touch a object with $0.25 \mathrm{Nm}$ torque ten times. The position accuracy for a proximal joint with Dyneema tendons, with mixed Dyneema steel(steel extensors and Dyneema flexors) and for steel tendons is compared (Fig.: 14). Although the position of joint is calculated on the one side from flexor and extensor tendon and on the other side only from the extensors. As expect the errors are quite big for uncompensated tendon elongation because the FAS are calibrated for Dyneema and for the tendon length in the forearm. It is better to calibrated the elongation with mounted fingers to get the real tendon length. If the joint position is calculated from both tendon, the creeping error is $1.3^{\circ}$ for Dyneema and $0.3^{\circ}$ for steel after five minutes. The elongation of the steel comes from the glued splices and should be replaced by crimped terminals. If it calculates from the extensors, the results are improved for all solutions, at which the calibration error of the FAS can have more influence. Best solution is the use of a link side sensor for initial position and then calculated the link position by the higher resolution motor and FAS sensors.

\section{Video Explanation: Robustness and under actuated hand mode}

To show again that the fingers are still robust against collisions, the finger is hit by a hammer and then grasps an object in the under actuated mode. In this mode the finger 


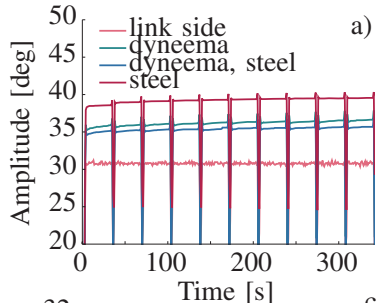

a) $\begin{array}{r}50 \\ 45\end{array}$
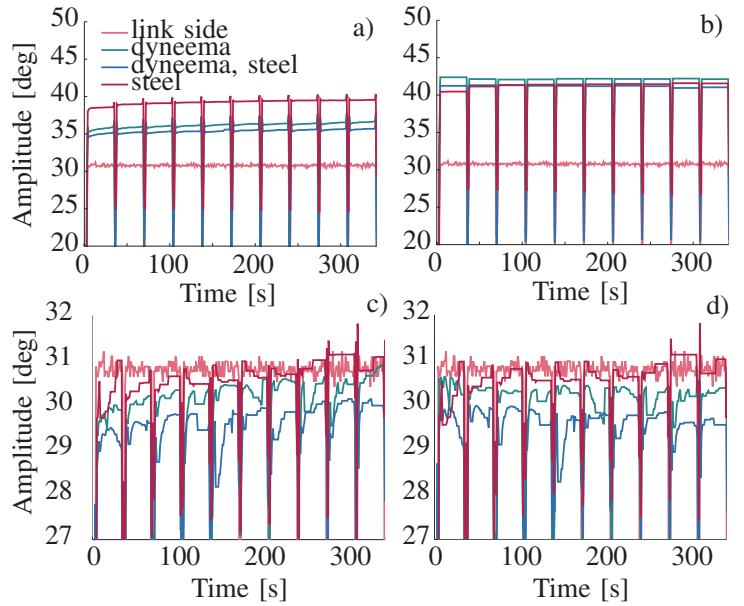

Fig. 14: (a) uncompensated elongation $q_{\text {close }}$ from two tendons, (b) uncompensated elongation $q_{\text {close }}$ from extensor tendon (c) compensated elongation $q_{\text {close }}$ from two tendons, (d) compensated elongation $q_{\text {close }}$ from extensor

behavior is quite like a one tendon finger. The first joints drives until it reached an adjusted torque or the adjusted end position. Then comes the next and so on. The minimum fingertip grip force without friction compensating is $0.8 \mathrm{~N}$. It is advantageous that the objects can be gripped without planning. The results are shown in a short video. Also a weight of $19 \mathrm{~N}$ is lifted by the finger to demonstrate his performance. 12

\section{CONCLUSION}

The new finger design for the Awiwi hand is introduced in this paper. It reduces the friction by about $20 \%$. The accuracy could be improved to yield a positioning error below $2^{\circ}$ in each joint. Furthermore, it shows that the more robust fiber tendons like Dyneema bring a lot bending friction on when using ball bearing pulleys. With the link side position lost through creep a change to the steel tendon brings a more reliable system. Different solutions for the link side measurements are considered in this paper. A very compact link side sensor is developed based on a Hall effect sensor.

The next step is to test the new fingers for grasping and manipulation. Although a new palm is in development for better maintenance. New grip surface of the finger are developed for better friction and larger contact surfaces. Thereby the human has to be analyzed, to get a better understanding. The test finger will be tested in $\mathrm{N}+1$ configuration and then compared with the two other solution.

\section{ACKNOWLEDGMENTS}

This work has been funded by the European Commission Seventh Framework Program as part of the project The Hand Embodied (grant no. 248587).

\footnotetext{
${ }^{12}$ Highspeed movements are not shown, because the maximum velocity is mostly depending from the actuators, which still allow joint speed above 720 degree/s.
}

\section{REFERENCES}

[1] L. Rybok, S. Friedberger, U. Hanebeck, and R. Stiefelhagen, "The KIT Robo-kitchen data set for the evaluation of view-based activity recognition systems," in Humanoid Robots, IEEE/RAS International Conference on, 2011, pp. 128-133.

[2] M. Raibert, "Dynamic legged robots for rough terrain," Humanoid Robots (Humanoids), 2010 10th IEEE-RAS International Conference on, 2010.

[3] S. Kawatsuma, M. Fukushima, and T. Okada, "Emergency response by robots to Fukushima-Daiichi accident: summary and lessons learned," Industrial Robot: An International Journal, vol. 39, pp. 428-435, 2012.

[4] M. A. Diftler and al., "Robonaut 2 - the first humanoid robot in space," Robots and Automation, IEEE/RSJ International Conference on, pp. 2178-2183, 2011.

[5] M. Grebenstein and al., "The DLR hand arm system," Robotics and Automation(ICRA), IEEE International Conference on, pp. 3175 $3182,2011$.

[6] M. G. Catalano, G.Grioli, A. Serio, E. Farnioli, C.Piazza1, and A. Bicchi, "Adaptive synergies for a humanoid robot hand," Humanoid Robots, IEEE/RSJ International Conference on, pp. 1251-1 $\overline{256,2012 .}$

[7] A. Dollar and R. Howe, "Simple, reliable robotic grasping for human environments," Technologies for Practical Robot Applications, IEEE International Conference on, pp. 156-161, 2008.

[8] M. Grebenstein, M. Chalon, G. Hirzinger, and R. Siegwart, "Antagonistically driven finger design for the anthropomorphic DLR hand arm system," Humanoid Robots, IEEE/RAS International Conference on, pp. 609-616, 2010.

[9] M. Grebenstein, M. Chalon, W. Friedl, S. Haddadin, T. Wimböck, G. Hirzinger, and R. Siegwart, "The hand of the DLR Hand Arm System: Designed for interaction," Robotics Research, International Journal on. Special Issue: Towards Autonomous Physical Human-Robot Interaction, vol. 31, no. 13, pp. 1531-1555, 2012.

[10] E. Ackerman, "Sandia National Labs Gives Roboticists a Hand," http://spectrum.ieee.org/automaton/robotics/humanoids/ sandia-labs-robotic-hand-, Aug. 2012.

[11] Sandia National Laboratories, "Lifelike, cost-effective robotic Sandia Hand can disable IEDs," https://share.sandia.gov/news/resources/ news_releases/robotic_hand/, Aug. 2012.

[12] M. R. Cutkosky, "On grasp choice, grasp models, and the design of hands for manufacturing tasks," Robotics and Automation, IEEE Transactions on, vol. 5, no. 3, pp. 269-279, 1989.

[13] T. Feix, H.-B. Schmiedmayer, J. Romero, and D. Kragić, "A comprehensive grasp taxonomy," Robotics: Science and Systems V; Workshop on Understanding Human Hand for Advancing Robotic Manipulation, 2009.

[14] M. Grebenstein, Approaching human performance: The functionality driven Awiwi robot hand (Springer Tracts in Advanced Robotics). Springer, 2012. [Online]. Available: http://www.amazon.com/ Approaching-Human-Performance-Functionality-Driven-Springer/dp/ 3319035924\%3FSubscriptionId\%3D0JYN1NVW651KCA56C102\% 26tag\%3Dtechkie-20\%26linkCode\%3Dxm2\%26camp\%3D2025\% 26creative\%3D165953\%26creativeASIN\%3D3319035924

[15] W. Friedl, M. Chalon, J. Reinecke, and M. Grebenstein, "FAS a flexible antagonistic spring element for a high performance over actuated hand," Intelligent Robots and Systems, IEEE/RSJ International Conference on, pp. 1366-1372, 2011.

[16] L. Birglen, T. Laliberté, and C. Gosselin, Underactuated robotic hands, ser. Springer Tracts in Advanced Robotics, 2008, vol. 40.

[17] J. Reinecke, M. Chalon, W. Friedl, and M. Grebenstein, "Guiding effects and friction modeling for tendon driven systems," Robotics and Automation (ICRA), 2014 IEEE International Conference on, pp. 6726-6732, 2014.

[18] R. Ozawa, K. Hashirii, and H. Kobayashi, "Design and control of underactuated tendon-driven mechanisms," Robotics and Automation, IEEE International Conference on, pp. 1522-1527, 2009.

[19] G. Palli, U. Scarcia, C. Melchiorri, and G. Vassura, "Development of robotic hands: The UB hand evolution," Intelligent Robots and Systems, International Conference on, pp. 5456-5457, 2012.

[20] A. Cavallo, G. D. Maria, C. Natale, and S. Pirozzi, "Optoelectronic joint angular sensor for robotic fingers," Sensors and Actuators A: Physical, vol. A152, pp. 203-210, 2009.

[21] M. Grebenstein, M. Chalon, G. Hirzinger, and R. Siegwart, "A method for hand kinematics designers," 1st International Conference on Applied Bionics and Biomechanics, 2010. 